\title{
Inner ear defects and hearing loss in mice lacking the collagen receptor DDR1
}

\author{
Angela M Meyer zum Gottesberge ${ }^{1}$, Oliver Gross², Ursula Becker-Lendzian' ${ }^{1}$, Thomas Massing ${ }^{1}$ and \\ Wolfgang $\mathrm{F} \mathrm{Vogel}^{3}$
}

Discoidin domain receptor 1 (DDR1) is a tyrosine kinase receptor that is activated by native collagen. The physiological functions of DDR1 include matrix homeostasis and cell growth, adhesion, branching, and migration, but the specific role of DDR1 in the development and function of the inner ear has not been analyzed. Here, we show that deletion of the DDR1 gene in mouse is associated with a severe decrease in auditory function and substantial structural alterations in the inner ear. Immunohistochemical analysis demonstrated DDR1 expression in several locations in the cochlea, mostly associated with basement membrane and fibrillar collagens; in particular in basal cells of the stria vascularis, type III fibrocytes, and cells lining the basilar membrane of the organ of Corti. In the stria vascularis, loss of DDR1 function resulted in altered morphology of the basal cells and accumulation of electron-dense matrix within the strial epithelial layer in conjunction with a focal and progressive deterioration of strial cells. Cell types in proximity to the basilar membrane, such as Claudius', inner and outer sulcus cells, also showed marked ultrastructural alterations. Changes in the organ of Corti, such as deterioration of the supporting cells, specifically the outer hair cells, Deiters', Hensen's and bordering cells, are likely to interfere with mechanical properties of the organ and may be responsible for the hearing loss observed in DDR1-null mice. These findings may also have relevance to the role of DDR1 in other disease processes, for example, those affecting the kidney.

Laboratory Investigation (2008) 88, 27-37; doi:10.1038/labinvest.3700692; published online 19 November 2007

KEYWORDS: inner ear; stria vascularis; DDR1; Discoidin domain; hearing loss; collagen

The inner ear is rich in extracellular matrix (ECM), which plays an essential role in the elasticity and rigidity of the structures that sustain the function of the cochlea during sound reception and transformation into electrical signals. Collagens are major constituents of the ECM and expression of collagen types I, II, III, IV, V, IX, and XI in the inner ear has previously been reported. ${ }^{1-3}$ Among these, collagen II has been detected in the mammalian tectorial membrane, spiral limbus, spiral ligament, and as a component of the basilar membrane. ${ }^{1,4,5}$ Type $\mathrm{V}$ collagen may be associated with collagen II and IX, but was also identified in the strial capillary basement membrane (BM) associated with collagen IV (AMMzG, unpublished observation). BMs form a continuous structural network reaching from Reissner's membrane over the dental cells, inner sulcus, and basilar membrane to the outer sulcus and the root cells. An extensive network of BMs is also associated with the spiral ganglion cells and their associated axons as well as with the vessels of the inner ear. ${ }^{2,6}$

In the cochlea, three distinct types of epithelia surround the endolymphatic compartment: the sensory epithelium (eg organ of Corti), the ion transporting epithelium (eg the stria vascularis), and a third, unspecialized epithelial layer (reviewed by Raphael and Altschuler ${ }^{7}$ ). While the organ of Corti is composed of various types of sensory hair cells and accessory supporting cells, the stria vascularis is a complex tissue composed of several different cell types. These include marginal cells (MC), which line the lumen of the cochlear duct and are of ectodermal origin; basal cells (BC), which form a continuous sheet-like layer, are coupled to one another by multiple tight junctions ${ }^{8}$ and are suggested to be of mesodermal origin; ${ }^{9,10}$ and intermediate cells (IMC), which are melanocyte-like cells. IMC are presumably derived from the neural $\mathrm{crest}^{11}$ and are interspersed between the MC and

\footnotetext{
${ }^{1}$ Department of Otorhinolaryngology, University of Duesseldorf, Duesseldorf, Germany; ${ }^{2}$ Department of Nephrology and Rheumatology, Goettingen University, Goettingen, Germany and ${ }^{3}$ Department of Laboratory Medicine and Pathobiology, University of Toronto, Toronto, ON, Canada Correspondence: Dr WF Vogel, PhD, Medical Sciences Building, Room 6342, 1 King's College Circle, Toronto, ON, Canada M5S 1 A8.

E-mail:w.vogel@utoronto.ca

Received 5 July 2007; revised 1 October 2007; accepted 6 October 2007
} 
BC layers. In the adult stria, the MC form extensive interdigitations with the BC and IMC. The less-specialized epithelia, Reissner's membrane and cells lining the outer and inner sulcus, are thought to form permeability barriers separating fluid spaces, however their exact function is not fully defined. ${ }^{12}$ In addition, the stria vascularis contains a rich supply of blood vessels, which are required to sustain the energy-consuming ion transport that maintains endolymph composition and endocochlear potential, functions essential for acoustic signal transduction. ${ }^{13,14}$

Clinical and experimental studies in normal and diseased organisms have established a strong relationship between the kidney and the inner ear. As a consequence of analogous roles in electrolyte balance, several cell types in these two organs possess similar morphological and ultrastructural features, and a number of highly related transporter proteins, ion channels, and BM proteins are found in the kidney and inner ear. Moreover, drug intervention (nephrotoxicity, ototoxicity) or genetic predisposition can result in correlative disturbances in the function of the inner ear and kidney (reviewed by Izzedine $e t a l^{15}$ ). For example, mutations in the $\alpha 3, \alpha 4$, or $\alpha 5$ chain of type IV collagen causes Alport syndrome, which is characterized by renal insufficiency. ${ }^{16}$ The most common extra-renal manifestation of Alport syndrome is deafness, occurring in approximately $80 \%$ of affected males. The inner ear phenotypes of mice and dogs with Alport-like type IV collagen mutations have been described in detail. ${ }^{17,18}$

Currently, two main classes of collagen-binding receptors are known: the integrins, which are heterodimers formed between $\beta 1$ and $\alpha 1,-2,-10$, or -11 subunits, and the Discoidin domain receptors (DDRs). ${ }^{19}$ The mammalian genome contains two DDR genes, DDR1 (previously also known as NEP, Cak, TrkE, MCK-10, EDDR, or NTRK4) and DDR2 (CCK-2, Tyro10, or TKT). The DDRs are distinguished from other receptor tyrosine kinases by the presence of an extracellular domain homologous to discoidin I, a lectin originally identified in the slime mold Dictyostelium discoideum. The DDR1 protein is widely expressed in normal and transformed cells and is activated by various types of collagen, including types I-VI, VIII, and X..$^{20,21}$ In early development, DDR1 is found predominantly in neuroectodermal cells, whereas in adult tissues it is expressed in epithelial cells, particularly in the mammary gland, brain, lung, kidney, and gastrointestinal tract, as well as in vascular smooth muscle cells, oligodendrocytes, and macrophages. ${ }^{22}$ Aberrant expression and signaling of DDR1 has been implicated in several human diseases associated with accelerated matrix turnover, including tumor invasion, atherosclerosis, and tissue fibrosis. ${ }^{23-25}$

The generation of DDR1-null mice by gene targeting produced viable animals that were smaller than control littermates. ${ }^{26}$ A percentage of mutant females were unable to bear offspring due to impaired blastocyst implantation, and when implantation did occur the mutant females were unable to lactate. Histological analysis showed that the alveolar epithelium failed to secrete milk proteins into the lumen of the mammary gland. This lactational defect appears to be caused by a cell-autonomous defect within the epithelial compartment. ${ }^{27}$ Furthermore, the absence of DDR1 leads to alterations of the glomerular basement membrane in the kidney ${ }^{28}$ and altered responses in a glomerulosclerosis model. ${ }^{29}$ On the basis of these studies, we hypothesized that DDR deficiency may also affect the function of the inner ear. To analyze the role of DDR1 in auditory function, we conducted functional and morphological studies of the cochlea from DDR1-knockout mice at the ages of 1, 2, and 4-5 months.

Here, we report a severe hearing loss in DDR1-null mice associated with focal and progressive alterations in the stria vascularis that are accompanied by accumulation of electrondense compounds within the epithelial layer and by pathological changes in several cell types lining the basilar membrane. We also present immunolocalization data showing the cellular distribution of DDR1 in the cochlear duct.

\section{MATERIALS AND METHODS \\ Mice}

Breeding of 129/Sv mice heterozygous for DDR1 produced wild-type, heterozygote, and homozygote mice in a Mendelian ratio. ${ }^{26}$ Mice were genotyped after weaning and animals with different genotypes from the same litter were used for our analysis. ${ }^{28}$ The cochlea from DDR $1-/-$, DDR $1+/-$, and DDR $1+/+$ mice aged 4 weeks $(n=3), 8$ weeks $(n=31)$, and $16-18$ weeks $(n=16)$ were processed and evaluated by transmission electron microscopy using a previously described procedure ${ }^{30}$ or immunohistochemically by light microscopy. Auditory function was evaluated in animals aged 2 months and 4-5 months. All animal procedures were performed in accordance with protocols approved by the local animal care committees of the German and Canadian authorities.

\section{Auditory Brainstem Response}

Auditory evoked brainstem response (ABR) was measured in mice aged 8 weeks and 16-18 weeks from each group. The number of animals per group is given in Figure 1. Mice were anesthetized by intraperitoneal injection of ketamine $(100 \mathrm{mg} / \mathrm{kg})$ and xylazine $(5 \mathrm{mg} / \mathrm{kg})$. Needle electrodes were placed under the skin at the vertex and medial of the right and left ears and a ground electrode was placed in the midline behind the supraorbital tori. Impedance of the electrodes was $3.5 \mathrm{k} \Omega$ at the maximum. A specific hardware/software combination (ZLE-System Technik, Munich, Germany) was used to induce the stimuli via a loudspeaker located at a distance of approximately $4 \mathrm{~cm}$ from the acoustic axis of the pinna. The external auricular canal of the other ear was plugged with an adhesive agent. Ninety microsecond pulses (frequency range $5-15 \mathrm{kHz}$ ) were delivered at sound pressure levels ranging from 25 to $100 \mathrm{~dB}$ in 5 -dB steps. ABRs were based on the average of 1000 responses at each sound 


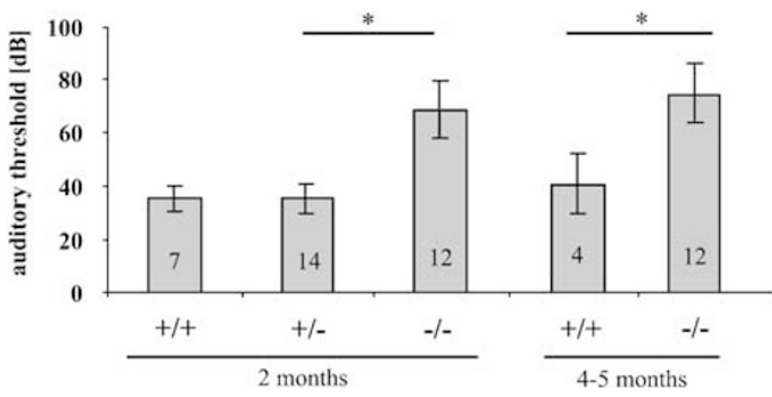

Figure 1 Loss of hearing in DDR1-null mice. Auditory thresholds (mean \pm s.d.) of wild-type (DDR1 $+/+$ ), heterozygote (DDR $1+/-$ ), and homozygote knockout mice (DDR1-/-) were determined at 2 months and 4-5 months using click evoked auditory brainstem potentials. The number of animals per group is given in each bar; the asterisks indicate statistical significance $(P<0.05)$.

pressure level. Auditory thresholds were based on visually detectable amplitude from a reproducible ABR waveform. All data were analyzed for group arithmetic means and standard deviations. Student's unpaired $t$-test was used to compare the ABR threshold of each group, with $P<0.05$ defined as the level of statistical significance.

\section{Tissue Preparation}

After ABR measurements, the anesthetized animals were perfused intracardially with buffer (isotonic $\mathrm{NaCl}$ and heparin, $\mathrm{pH} 7.3$ ), followed by $2 \%$ paraformaldehyde in PBS (PFA). After $5 \mathrm{~min}$ of perfusion, the temporal bones were removed, the cochleae were opened in the apex, and one ear was gently perfused through the oval window with EM fixative (2\% PFA, 2\% glutaraldehyde in PBS) and the other with $4 \%$ PFA. Both ears were exposed to fixative for $24 \mathrm{~h}$ at $4{ }^{\circ} \mathrm{C}$. Temporal bones were decalcified using $10 \%$ EDTA in PBS at room temperature for 3-4 days with daily changes of the decalcification solution.

\section{Electron Microscopy}

The temporal bones were dissected, and the inner ear was divided at the round window region into two parts: the cochlea and the vestibular organ. These were post-fixed with $1 \%$ phosphate-buffered $\mathrm{OsO}_{4}$ for $1 \mathrm{~h}$ at room temperature, dehydrated in a graded ethanol series, block stained with uranyl acetate in $70 \%$ ethanol followed by a propylene oxide series, and embedded in EPON epoxy resin. Ultrathin sections (100-200 nm) were stained with uranyl acetate and lead citrate, and examined with a Hitachi H-6000 electron microscope.

\section{Immunohistochemistry}

Paraffin sections $(5 \mu \mathrm{m})$ were deparaffinized in xylene and rehydrated using graded ethanol steps, followed by immersion in PBS for $15 \mathrm{~min}$. For peroxidase and DAB detection, sections were incubated in $10 \% \mathrm{H}_{2} \mathrm{O}_{2}$ for $30 \mathrm{~min}$ to quench endogenous peroxidase activity. The sections were pretreated with $0.1 \%$ trypsin (Sigma) in PBS for $15 \mathrm{~min}$ at room temperature. Slides were blocked for $40 \mathrm{~min}$ in $5 \%$ low-fat milk and 5\% serum of secondary antibody source in PBS containing $0.1 \%$ Triton-X100 (PBST). DDR1 polyclonal antibodies (sc-532, raised in rabbit against an epitope mapping to the C-terminus of DDR1; or sc-7553, raised in goat against an epitope mapping the N-terminus of DDR1; Santa Cruz Biotechnology) were diluted 1:25 in PBST containing $1 \%$ BSA and applied to tissue sections at room temperature overnight. Following application of primary antibody, the sections were rinsed twice with PBST for $5 \mathrm{~min}$ and incubated with the appropriate secondary antibody; either HRP-conjugated rabbit anti-goat $\operatorname{IgG}(1: 100,1 \mathrm{~h})$, or Alexa488-conjugated goat anti-rabbit $\operatorname{IgG}(1: 100,2 \mathrm{~h})$. The sections were rinsed, mounted with Immunomount (Shandon), and evaluated by fluorescence microscopy (Olympus BX 40).

\section{RESULTS \\ DDR1-Null Mice Develop a Profound Hearing Deficit by 2 Months of Age}

To assess hearing function in DDR1-null and control mice, ABR measurements were recorded in groups of knockout (DDR1-/-), heterozygote (DDR $1+/-$ ), and wild-type $(\mathrm{DDR} 1+/+)$ mice, and the mean threshold level for each group was determined (Figure 1). ABRs of control animals were indicative of mature auditory function: the threshold, latency, and waveforms of evoked potentials were within the normal range of adult mice. At a frequency range of $5-15 \mathrm{kHz}$, a severe loss of auditory function was detected in 2-month-old DDR1-/- mice: the auditory threshold $(75 \mathrm{~dB})$ in homozygote mice was significantly higher $(P<0.0001)$ than that of wild-type or heterozygote littermates $(35 \mathrm{~dB})$. In contrast, there was no significant difference between heterozygote and wild-type mice. A significant difference between the knockout and wild-type group $(P<0.001)$ was also observed in mice aged $4-5$ months.

\section{DDR1 Expression in the Cochlea}

The drastic loss of auditory function in DDR1-null mice suggests an important role of this gene in the function of the inner ear. We therefore assessed DDR1 expression in the mouse inner ear by immunofluorescence microscopy. Figure 2A shows a cross-section of the cochlea from an adult wildtype mouse. This facilitates localization of various anatomical features, and demonstrates that wild-type tissue remains intact following fixation. For analysis of DDR1 expression, we used two different antibodies raised against epitopes mapping to either the C- or N-terminus of DDR1, which yielded nearly overlapping staining with only minor differences in staining intensities. When adjacent sections were stained with a DDR1-specific antibody, DDR1 was distributed mainly in the lateral wall and in cells lining the basilar membrane, including the organ of Corti (Figure 2B-E). In the lateral wall, positive immunostaining was observed in type III fibrocytes and vessels of the spiral ligament, in the strial basal cells, and in focal areas within the stria layer (Figure $2 \mathrm{~B}-\mathrm{D}$ ). There was 

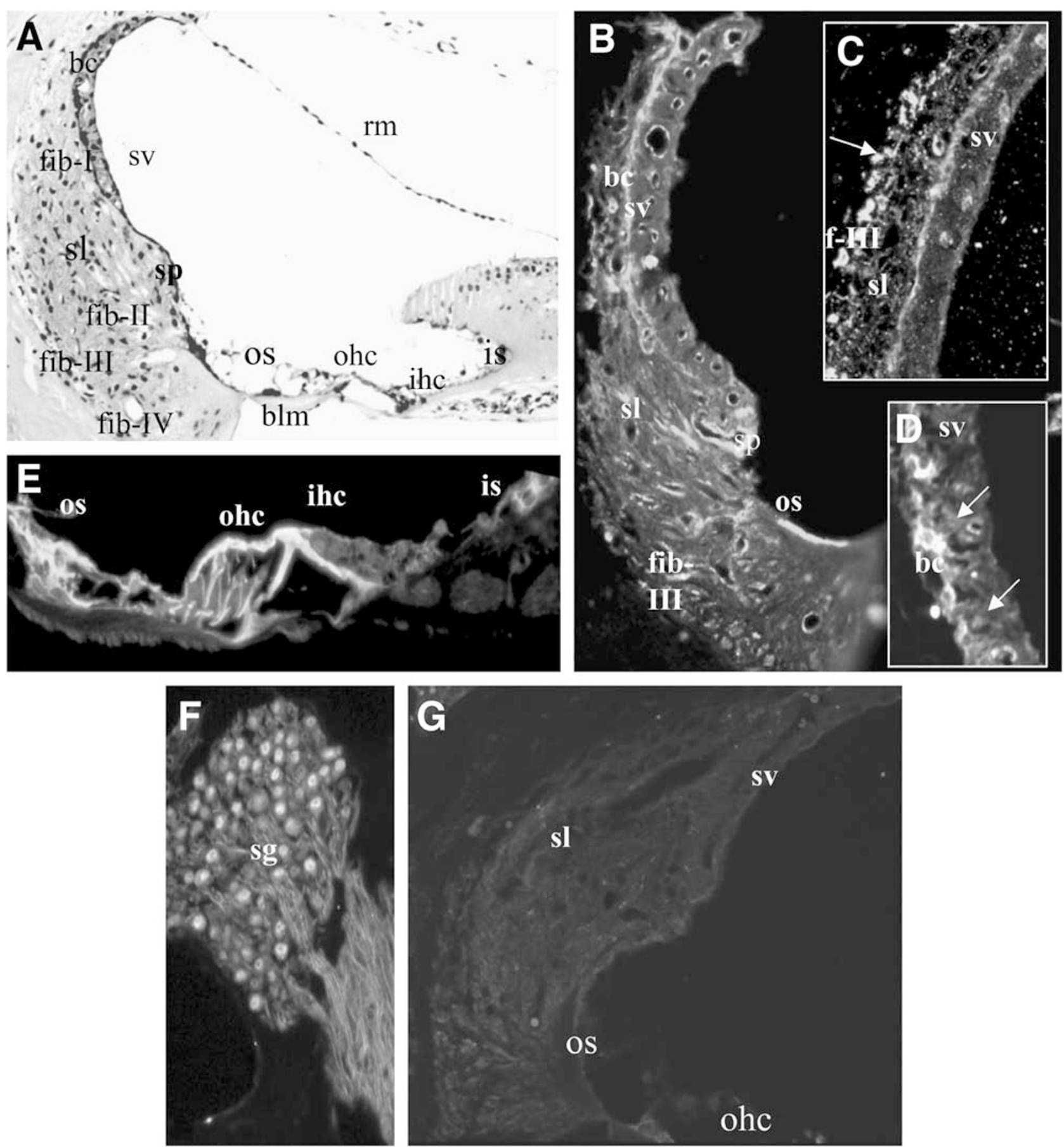

Figure 2 Expression of DDR1 in the cochlea. (A) Midmodiolar section of the cochlear duct at the basal turn from a wild-type mouse (2 months old) stained with Masson Goldner trichrom. Relevant morphological details are indicated. (B-G) Expression of DDR1 in the inner ear was analyzed by immunofluorescence in wild-type (B-F) and DDR1-null mice (G). Images show the lateral wall of the basal turn (B, D), the lateral wall of the hook (C), the basilar membrane including the organ of Corti and adjacent inner and outer sulcus (E), and the spiral ganglion (F). In the lateral wall, DDR1-positive cells were found mainly in the area of type-III fibrocytes of the spiral ligament adjacent to the bony wall (arrows in C) and in the basal compartment of the stria vascularis. Lower levels of expression were observed within the strial epithelium (arrows in D) and in cochlear vessels. Individual cells of the outer sulcus, the inner sulcus, the organ of Corti, Deiters' cells, phalangial processes, and the cuticular plate (part of the outer hair cell) were prominently stained, whereas the cell body of the outer hair cells $(\mathbf{E})$ and the spiral ganglion $(\mathbf{F})$ showed weak immunoreactivity. No immunostaining was observed in a section of DDR1-null mice (G). Abbreviations: sv, stria vascularis; sl, spiral ligament; os, outer sulcus; is, inner sulcus; rm, Reissner's membrane; fib-I, II, III, IV, type-I, -II, -III, -IV fibrocytes; bc, basal cell; ohc, outer hair cell; ihc, inner hair cell; sg, spiral ganglion; blm, basilar membrane; mc, marginal cell. Magnification: $(\mathbf{A}) \times 150$; (B, C, E, F and G) $\times 250$; (D) $\times 460$. 

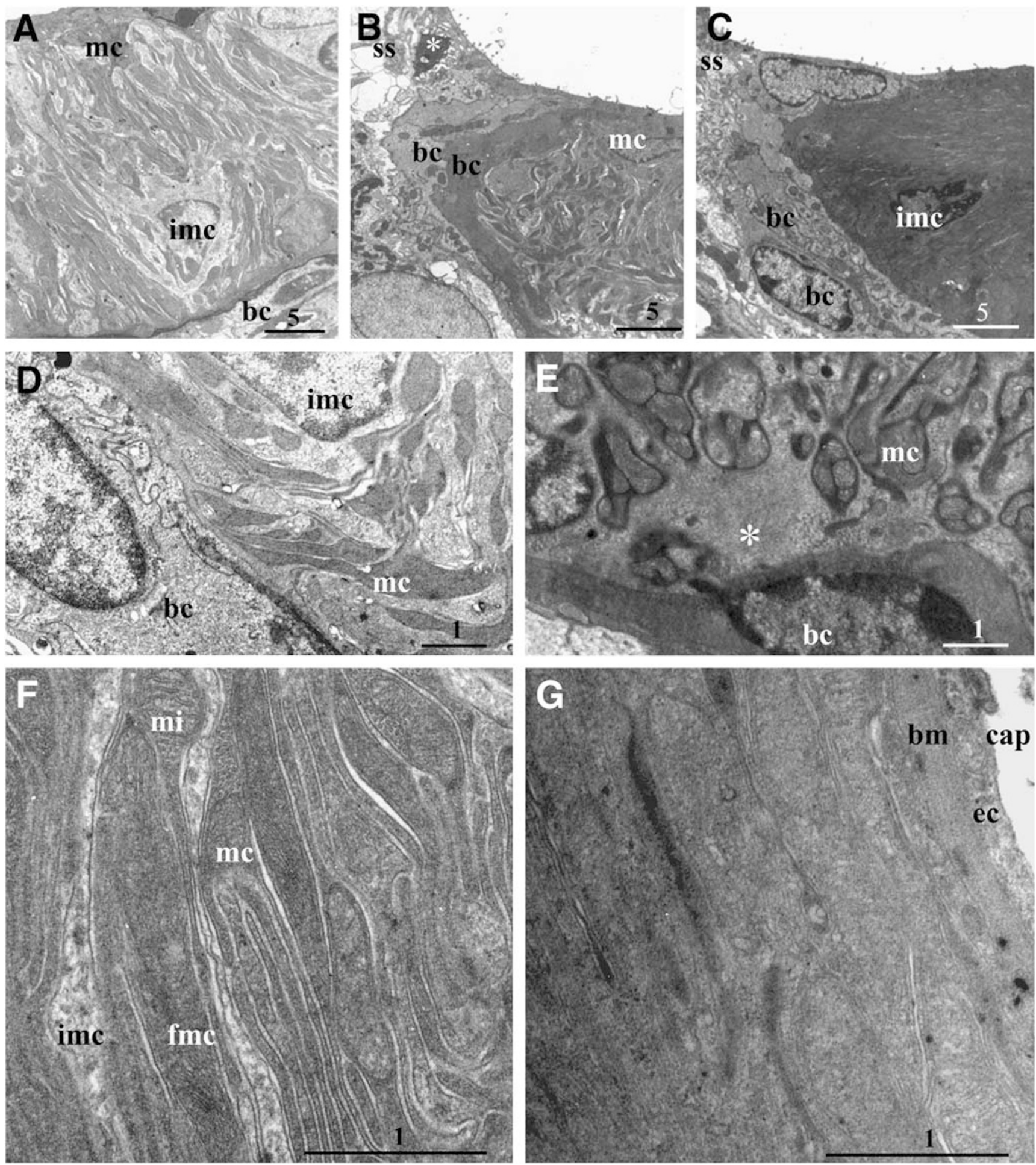

Figure 3 Ultrastructural changes of the stria vascularis in 2-month-old DDR1-null mice. Transmission electron microscopy of tissue from wild-type (A, D, and F) and DDR1-null (B, C, E, and G) mice. (A, B, and C) Compared with wild-type animals (A), the stria vascularis in knockout mice (B, C) was characterized by aberrant morphology of the basal cells. The asterisk in (B) indicates a pyknotic suprastrial cell. (D, E) Amorphous matrix (asterisk in E) is deposited around the basal infoldings of the marginal cells in the knockout (E), but not in control animals (D). (F, G) Higher magnification of the stria vascularis demonstrates a loss of characteristic spaces between the marginal and intermediate cell infoldings in the DDR1-null (G) compared with control tissue (F). Abbreviations: bc, basal cells; mc, marginal cells; imc, intermediate cells; cap, capillary; ec, endothelial cell; bm, basal membrane. Scale bars are in $\mu \mathrm{m}$. 
discrete labeling of cells in the outer sulcus, the inner sulcus, Claudius' cells, and cells bordering the outer hair cells (Figure 2E). In the organ of Corti, the pillar cells, the processes of the phalangial cells, and Deiters' cells were strongly stained, while lower reactivity was found proximal to the cell body of the outer hair cell (Figure 2E). In spiral ganglion cells, a modest immunoreactivity was observed in the Schwann cell-neuron complex (Figure 2F). Staining was absent when immunohistochemistry was performed on sections from DDR1null mice and when non-immune serum was used instead of the primary anti-DDR 1 antibody, confirming the specificity of this antibody (Figure $2 \mathrm{G}$ ).

\section{Morphological Characterization of the Stria Vascularis in DDR1-Null Mice}

Next, we compared the inner ear morphology of wild-type, heterozygote, and DDR1-null mice. We initially focused on the stria vascularis because of the prominent expression of DDR1 in this region. Heterozygote mice were indistinguishable from wild type with respect to gross morphology (data not shown). In contrast, a number of distinct alterations were observed in DDR1-knockout mice. In 1- and 2-month-old knockout mice, we observed activation of the BC associated with a substantial increase in ECM deposition within the stria vascularis. These ultrastructural alterations were distributed along the entire stria vascularis and present in different stages of progression (Figure 3A, control; Figure $3 \mathrm{~B}$ and $\mathrm{C}$, knockout). The $\mathrm{BC}$, which are normally flat and form multiple layers, were larger in the knockout, showed varying electron density, and were occasionally compressed due to increased matrix deposition (Figure 3B). Similarly, the basal infoldings of the marginal cells in the knockout were surrounded by more electron-dense deposits compared with the wild type (Figure 3D, wild type and E, knockout). In sections from several animals, clear demarcation of cell borders or identification of cellular organelles was not feasible due to overloading with ECM proteins (Figure 3C). At higher magnification, the characteristic intercellular spaces between the MC and IMC of the stria vascularis were evident in the wild type (Figure 3F) but largely deteriorated in DDR1-null mice (Figure 3G). Furthermore, the capillary membrane was difficult to discern due to electron-dense deposition (Figure 3G).

Compared with 2-month-old mice, these structural alterations were even more severe in 4- to 5-month-old DDR1-null animals (Figure 4A-D), which showed advanced deterioration of the $\mathrm{BC}$ especially close to Reissner's membrane or the spiral prominence (Figure 4B). As observed in younger animals, there was a considerable variation in morphological alterations, both between animals and along the length of the cochlea. The micrographs in Figure $4 \mathrm{~A}, \mathrm{C}$ and D were obtained from the same DDR1-null cochlea and demonstrate the observed range of alterations from a nearly normal morphology (a), to disorganized cells (c), to widespread lesions (d). In such regions, a hydropic alteration of the MC and IMC and their cellular infoldings was observed. In particular, rounding and curling of the basal infoldings of the $\mathrm{MC}$ and loss of their basolateral orientation was evident (Figure 4E). Vesiculation, fragmentation, scrolling of the membranes, mitochondrial swelling, and cellular condensation were also observed (Figure 4F). In such affected areas, the BM of capillaries showed severe lamination and the capillary wall was occasionally collapsed (Figure 4G). In the lateral wall, these alterations were restricted to the stria vascularis and individual fibrocytes adjacent to bone, while the neighboring cells of the spiral ligament and the capillaries appeared unchanged. In several animals, an increase in collagen fiber accumulation between spiral ligament cells was observed (arrows in Figure 4H). Additionally, mesothelial cells located on the perilymphatic side of Reissner's membrane showed condensation of the cytoplasm (Figure 4I). We also observed marked exostosis in the cochlear bony wall of several knockout mice (data not shown).

\section{Profound Alterations in the Organ of Corti from DDR1-Null Mice}

In 2-month-old DDR1-null mutants, Deiters' cells had a condensed appearance (Figure 5A) and serial sections showed that a small number of cells were detached from the basilar membrane. The most prominent alterations in the organ of Corti were seen in the lateral wall of outer hair cells containing lateral cisternae, which was partly detached from the cell body (arrow in Figure 5B). The inner hair cells were not affected, and vacuoles in the synaptic region were only occasionally observed (data not shown). This detachment was observed in $40-50 \%$ of the outer hair cells in each older knockout animal, but not in the control group (Figure 5C). Individual cells such as Claudius', Hensen's, and bordering cells (Figure 5F-H) showed enhanced electron density and a marked condensed appearance compared with normal cells. The ultrastructure of several other cell types lining the basilar membrane and adjacent to the internal and external sulcus differed between knockout and wildtype tissue. At 4 months, alterations in the organ of Corti were similar to those in the previously examined 2-month-old knockout animals; however, border cells adjacent to the inner hair cells now showed a dense appearance, leading to degenerated inner hair cells (data not shown).

We also examined the spiral ganglion cells in knockout and control mice. Under light microscopy, the mutants displayed no overt changes in the density of the spiral ganglion cells and nerve fibers; however, using electron microscopy, we observed focal disturbances in the spiral ganglion area especially near the base of the cochlea. In such areas, lamination of the myelin sheets surrounding the cochlear neurons was evident (Figure 5D), but pronounced pathological areas with accumulation of ECM similar to that seen in the stria vascularis were not found. Moreover, in one of six DDR1null specimens, collagen fibers were severely altered, forming multiple aggregates within the connective tissue of the spiral limbus (Figure 5E). 

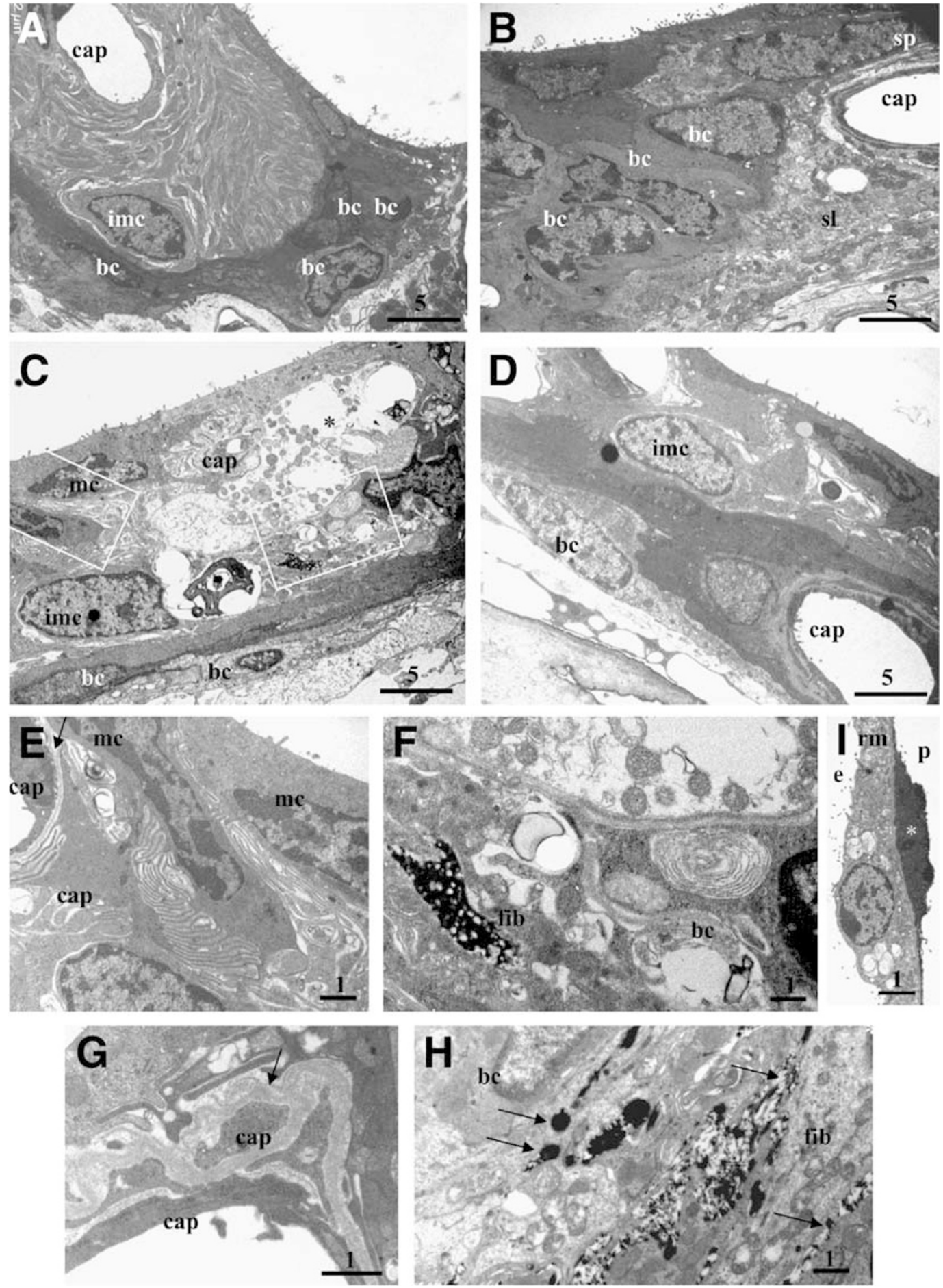

Figure 4 Ultrastructural changes of the stria vascularis in 4- to 5-month-old DDR1-null mice. (A, C, and D) Micrographs from different turns of the same DDR1-null cochlea demonstrating the range of alterations from a nearly normal morphology (A), to disorganized tissue architecture (C), to widespread lesions (D). The section from wild-type at 4-5 months was identical to the micrograph shown in Figure 3A. (B) Advanced modification of the BC adjacent to the spiral prominence. (E, F) Higher magnification of (C) showing cellular infoldings, mitochondrial swelling, vesiculation, membrane fragmentation, scroll-like membranous formation, and cellular condensation. (G) In several DDR1-null specimens, basket-like lamination (arrow) of the capillary basal membrane or collapsed capillaries were observed. (H) In the spiral ligament, disorganized and clustered collagen fibers (arrows) were present. (I) Condensed mesothelial cells facing the perilyphatic space were observed along Reissner's membrane. Abbreviations: bc, basal cells; mc, marginal cells; imc, intermediate cells; cap, capillary; sl, spiral ligament; fib, fibrocyte; rm, Reissner's membrane, e, endolymph; p, perilymph. Scale bars are in $\mu \mathrm{m}$. 

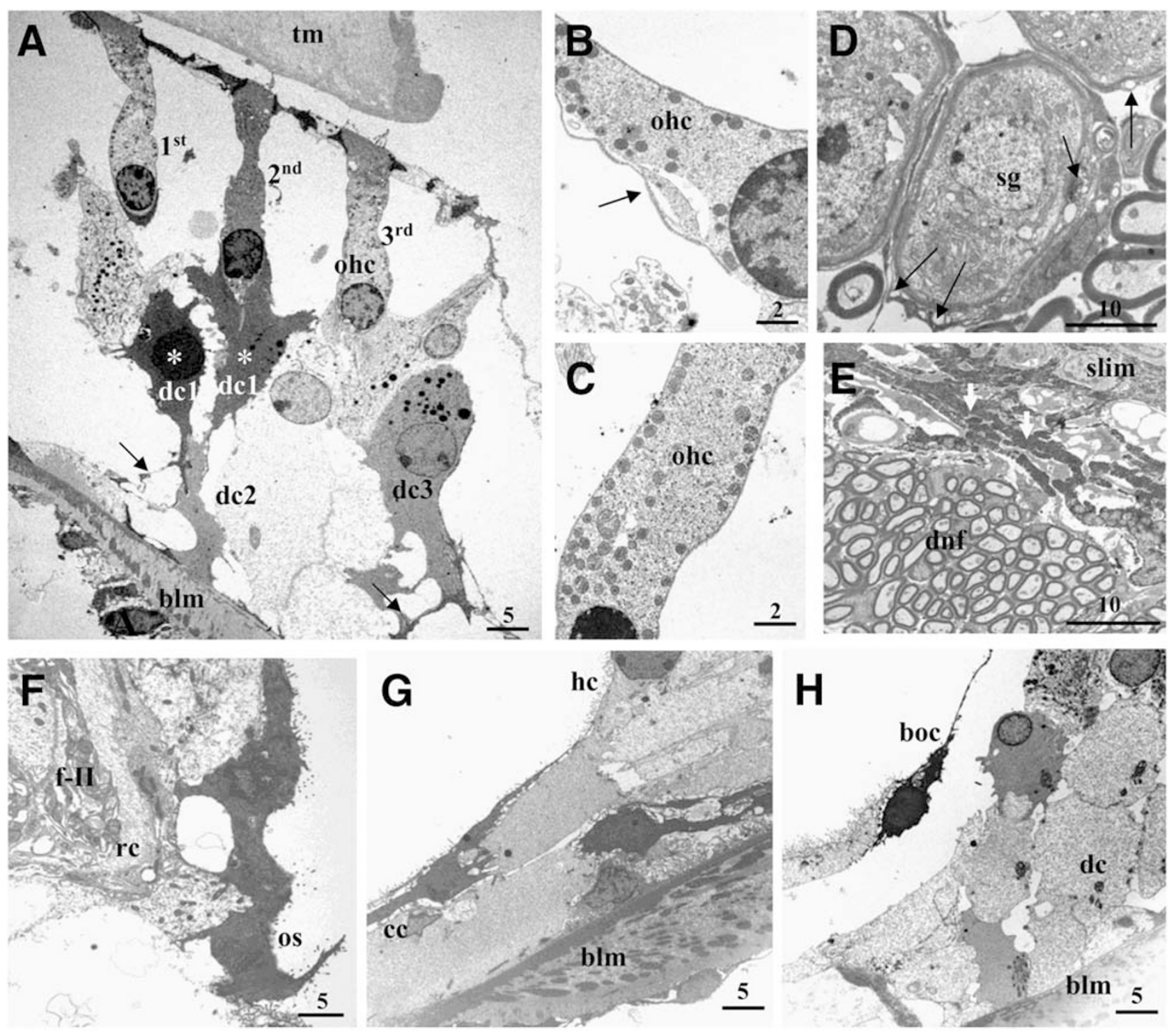

Figure 5 Morphological alterations of cells lining the basilar membrane, the organ of Corti, and the spiral ganglion in 4- to 5-month-old DDR1-null mice. (A) In the organ of Corti, the abnormal phenotype of DDR1-/- mice is restricted to the supporting cells, the bordering cells, and the outer hair cells (A, B), while the inner hair cells appeared unchanged. Individual Deiters' cells show variation of the electron density from dc1 with condensed appearance (asterisk in $\mathbf{A}$ ), to an intermediate stage in dc3, to normal appearance in dc2. Both dc1 and dc3 show altered attachment to the basilar membrane. Strikingly, the outer hair cells exhibited detachment from the cytoskeleton (arrow in B) compared with the wild-type control (C). (D) Excessive amounts of fibrous collagen were deposited adjacent to the area of nerve fibers of the spiral ganglion, forming irregular clumps within the connective tissue (arrows). (E) Spiral ganglion cells with focally perturbed myelinization were occasionally seen in all knockout animals (arrows). (F-H) Individual cells lining the outer sulcus (F) and basilar membrane were more electron-dense $(\mathbf{G}, \mathbf{H})$ than wild-type tissue. Abbreviations: ohc, outer hair cells; ihc, inner hair cell; dc, Deiters' cell; sg, spiral ganglion; slim, spiral limbus; tm, tectorial membrane; blm, basilar membrane; boc, bordering cell, cc, Claudius cell; hc, Hensens' cell. Scale bars are in $\mu \mathrm{m}$.

\section{DISCUSSION}

Molecular genetics and transgenic animals have provided powerful tools for the elucidation of the molecular basis of cochlear function and in recent years there has been remarkable progress in identifying genes that contribute to deafness. The data presented here identify the tyrosine kinase receptor DDR1 as an additional gene essential for inner ear function in the mouse. Loss of DDR1 function results in significantly decreased auditory function and affects several different cell types in the inner ear, as summarized in Figure 6. The majority of these cells are in close proximity to collagen, the cognate ligand for DDR1. The most pronounced pathological alterations were observed in the stria vascularis and in the organ of Corti. Based on our findings, we propose that DDR1 function is essential for maintaining tissue architecture and controlling collagen deposition in the inner ear of adult mice. DDR1-dependent adhesion signals may induce secretion of matrix metalloproteinases that promote matrix 


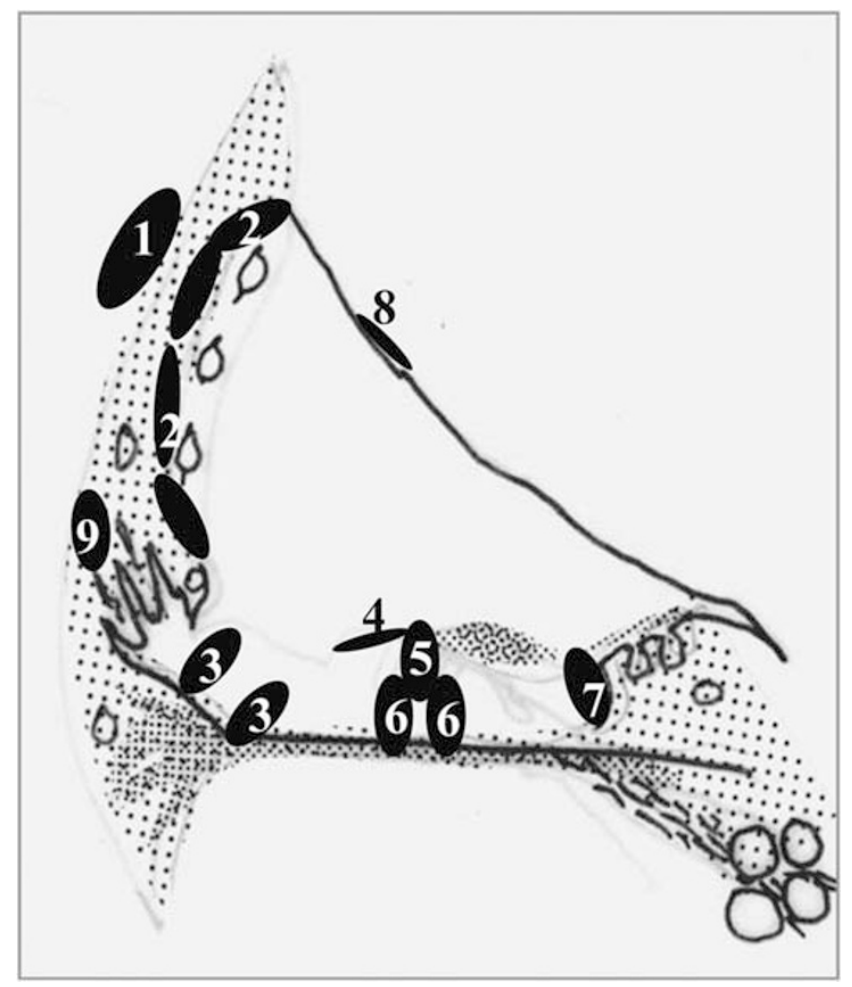

Figure 6 Schematic drawing of the cochlear duct highlighting specific regions with defects in DDR1-null mice. Fibrillar collagen is indicated by a dot matrix, and the basement membrane by dark lines. The numbers representing areas of altered morphology in DDR1-null mice are as follows: 1 , exostosis; 2 , basal cells; 3 , outer sulcus; 4 , border cells; 5 , outer hair cells; 6 , supporting cells; 7 ; inner sulcus; 8 , Reissner's membrane; 9 , spiral ligament.

breakdown and renewal. Loss of DDR1 adhesion signaling may lead to degeneration of the cell types identified in Figure 6 and enhanced accumulation of matrix molecules, as observed in this study. Additional research is necessary to identify the molecular nature of this ECM compound and to definitively link the severe progressive morphological alterations seen in older DDR1-null mice with the hearing loss observed in both young and old mice. In this regard, it will also be important to study alterations of the cochlea during embryonic and early postnatal development in the absence of DDR1; experimental protocols that were not available in this study.

DDR1 immunoreactivity was mainly located in the basal layer of the stria vascularis surrounding BC, and within the strial epithelial layer between the basal infoldings of the MC and dendrite-like projections of IMC. It has been suggested that these unique intercellular spaces are essential for extracellular fluid exchange between both cell types and for the generation of the endocochlear potential. ${ }^{31}$ Importantly, we show here that these spaces are reduced in DDR1-knockout mice. In adult animals (aged 2 months and 4-5 months), progressive pathological changes occurred with variability in the pattern of deterioration: in addition to variation in the degree of damage along the length of the cochlea, the spatial pattern of alterations was found to vary between animals. In control mice, $\mathrm{BC}$ form the lateral border of the stria vascularis and are flat overlapping cells that are joined by tight and gap junctions into a continuous cell layer. ${ }^{31}$ In contrast, the $\mathrm{BC}$ of DDR1-null animals were swollen, forming a thickened layer with accumulation of electron-dense matrix both within the cells and in the strial layer including the MC and IMC. The accumulation of homogenous dense matrix may lead to loss of the unique intra-strial spaces between the MC and IMC that are essential for the generation of the endocochlear potential. In 4- to 5-month-old DDR1-null mice, advanced deterioration of strial cells was evident as hydropic changes, mitochondrial swelling, vesiculation, membrane fragmentation, loss of the basolateral orientation of the infoldings, and thickening and lamination of capillary BM. Changes in the stria vascularis have been described in other animal models, for example after ototoxic insults or due to aging; however, these reports did not identify the specific site of primary pathology or uncover the molecular mechanism leading to such changes. The pathological alterations seen in our young adult DDR1 mutants, especially the abundant accumulation of homogenous granular material in the stria vascularis, differ from those described elsewhere when age and sequence of pathological alterations are taken into account. We failed to see any striking similarities between the degenerative pattern seen in DDR1-mutant mice and that observed following acoustical injury described earlier. ${ }^{32}$ Additionally, Spicer and Schulte ${ }^{33}$ described pathological changes due to presbycusis in aged (30-36 months old) gerbils. Although there are some similarities with respect to pathological alteration, their results differed from ours with regard to the timeline of events. In our DDR1 mutants, accumulation of the focally distributed dense material was evident from the start of our observations at 1 month of age (data not shown). The matrix was homogenously overloaded in the intra- and extracellular spaces leading to abundant accumulation surrounding the basal infoldings of MC. Furthermore, the accumulation of matrix was associated with a concomitant alteration of the $\mathrm{BC}$, which were unaltered in the gerbil study. ${ }^{33}$

Although it is well established that MC play a critical role in the generation of an ion gradient between the endolymph and perilymph, the function of strial BC has not been fully elucidated. Based on their morphological properties, it has been suggested that $\mathrm{BC}$ may function as a diffusion barrier between the perilymphatic and endolymphatic space. BC project slender processes towards the luminal surface, thereby influencing the movement of intra-strial fluid towards the lumen. ${ }^{10,34}$ This proposal is supported by the fact that BC express the GLUT1 transporter and are involved in the recycling of $\mathrm{K}^{+}$from the spiral ligament cells into the intrastrial spaces. ${ }^{35}$ The presence of vimentin and specific actin isoforms in $\mathrm{BC}$ confirms their origin from the mesodermal lineage and suggests a potential contractile function, analogous to myoepithelial cells. ${ }^{10}$ It seems likely that $\mathrm{BC}$ are involved in generation of the endocholear potential. 
The organ of Corti contains both sensory hair cells and supporting cells resting on the basilar membrane. In particular, Deiters' and pillar cells are thought to provide the structural support necessary to allow mechanical stimulation of hair cells in response to sound. Outer hair cells rest on Deiters' cells and have a cylindrical shape with the mitochondria mostly organized along the lateral wall. They contain contractile elements and their role as a cochlear amplifier is well established. ${ }^{12}$ DDR1-null mice exhibited both abnormal morphology of the supporting cells (Deiters', Hensen's, and bordering cells) and alteration of outer hair cells, and these changes may directly contribute to the threshold shift of the auditory function measured in 2month-old DDR1-null mice. In DDR1-null mice, Deiters' cells varied in intracellular electron density and cells with a condensed appearance tended to show reduced anchorage to the basilar membrane. These observations suggest that DDR1 may contribute to the attachment of the supporting cells to the basilar membrane. Loss of supporting cell rigidity in DDR1 mutants would result in decoupling of basilar membrane motion from tectorial membrane motion and loss of hearing acuity. The outer hair cells also showed severe alterations, with mitochondria largely disordered and the lateral membrane often detached from the cytoskeleton. Since a cellular motor responsible for the electromotile property of hair cells is located in the lateral wall (reviewed by Forge and Wright ${ }^{12}$ ), the observed alterations will likely compromise the function of outer hair cells resulting in an elevated hearing threshold as shown in these studies.

At present we cannot determine whether the defects observed in DDR1-null mice are primarily caused by the lack of cell attachment to basement membranes rich in type IV collagen, or whether the absence of DDR1 signaling initiates downstream pathways that lead to the transcriptional activation of ECM gene expression or induction of proteases to accelerate matrix turnover. Previous studies showed that DDR1 can inhibit collagen fibrillogenesis and disrupt the native structure of collagen fibers in vitro, suggesting that in the absence of DDR1, cells may upregulate collagen deposition. ${ }^{36}$ Conversely, the overexpression of DDR1 in human smooth muscle cells in vitro led to a reduction in collagen expression. ${ }^{37}$ In vivo, the loss of cell-matrix communication in DDR1-deficient podocytes appears to result in excess synthesis of basement membrane proteins, leading to disturbed anchorage of foot processes and disruption of the slit diaphragm. ${ }^{28,38}$ However, DDR1-null mice maintained sufficient renal function, indicating that the loss of hearing in these mice is not secondary to kidney failure. DDR2, which is activated by fibrillar collagens only, can potentially substitute for DDR1 function to some extent but very little is known about the overall redundancy of the two DDR family members. Interestingly, a search of the Morton Human fetal cochlea EST Databank yielded entries for both family members. ${ }^{39}$ At present, there are no known mutations of the $D D R 1$ gene in humans; however, DDR1 is located on chro- mosome $6 \mathrm{p} 21.3$ and lies within the recently described interval of the DFNB66 locus, which was linked to autosomal recessive nonsyndromic hearing loss in a large consanguineous family. ${ }^{40}$ It will be important to test whether DDR1 is mutated in this family. Additional work will also evaluate the role of DDR1 in other, nonhereditary, inner ear pathologies.

Collagen types I, II, III, V, IX, and XI, as well as the alpha 1-5 chains of type IV collagen, have been found in the inner ear. Germline mutations in several of these collagens have been linked to human syndromic and nonsyndromic deafness; for example, mutation of COL1A1 and COL1A2 cause osteogenesis imperfecta, and extensive skeletal alterations are regularly associated with hearing impairment. ${ }^{41}$ Mutations in COL9A1, COL2A1 and A2, COL11A1 and A2 are classified as various types of Stickler syndrome that manifest with ophthalmic, articular, orofacial, and auditory defects. ${ }^{42}$ Furthermore, mutations in the COL4A3-5 genes cause Alport syndrome, an inherited disorder showing progressive nephropathy, ocular abnormalities, and sensorineural hearing loss. ${ }^{43}$ In contrast, COL4A3-knockout mice did not present with an uniformly elevated hearing threshold, since only three out of 12 animals studied showed a significantly altered $\mathrm{ABR}^{44}$ In a parallel study, thickening of the strial BM was observed in COL4A3-null mice at the ultrastructural level; however, a slight shift in auditory threshold shift was reported only in some older animals. ${ }^{17}$ A study of the canine model of Alport syndrome revealed renal dysfunction very similar to that in the human syndrome. ${ }^{18}$ Strongest expression of canine COL4A3-5 chains was found in the spiral ligament as well as in a region adjacent to the bony wall that is populated by myofibrocytic cells (fibrocytes types III and IV) containing various contractile elements including actin, $\alpha$-actinin, non-muscle myosins, and tropomyosin. ${ }^{45,46}$ In this context, it is of interest that patients with specific mutations in $M Y H 9$, the gene coding for non-muscle myosin heavy chain IIA, also suffer from hearing loss. ${ }^{46,47}$ These mutations were originally described as Fechtner syndrome (DFNA17) but are now categorized within the MYH9 cohort. It is conceivable that certain collagens, DDR1 and related collagen receptors, and proteins in the actomyosin complex are acting in concert to maintain the tension of the inner ear and allow proper auditory signal transduction. Future studies will aim to characterize the exact role of DDR1 in such a complex.

In conclusion, we have identified the collagen receptor DDR1 as a surface molecule critically involved in the maintenance of the inner ear structure and function. We propose that a complex between triple helical collagen and DDR1 may function as one of the critical elements of mechanotransduction in the auditory system.

\section{ACKNOWLEDGEMENT}

We thank the staff from the animal house of the Center of Molecular Medicine Cologne (ZMMK, University of Cologne) for expert animal care. We thank O Goektas for technical assistance and SJ Harvey for critical reading of 
the manuscript. Parts of the present paper were previously published as abstracts at the annual meetings of the German and the American Societies of Otolaryngology in 2005 and 2006 (AMMzG) and at the annual meeting of the German and the American Societies of Nephrology in 2006 (OG). This work was funded by a grant from the Imhoff Foundation and the Deutsche Forschungsgemeinschaft (GR 1852/4-1) to OG. The support of the Canada Research Chair Program to WFV is acknowledged.

1. Asamura $K$, Abe $S$, Imamura $Y$, et al. Type IX collagen is crucial for normal hearing. Neuroscience 2005;132:493-500.

2. Cosgrove D, Samuelson G, Pinnt J. Immunohistochemical localization of basement membrane collagens and associated proteins in the murine cochlea. Hear Res 1996;97:54-65.

3. Tsuprun V, Santi P. Ultrastructural organization of proteoglycans and fibrillar matrix of the tectorial membrane. Hear Res 1997;110:107-118.

4. Dreiling FJ, Henson MM, Henson Jr OW. The presence and arrangement of type II collagen in the basilar membrane. Hear Res 2002;166:166-180.

5. Thalmann I. Collagen of accessory structures of organ of Corti. Connect Tissue Res 1993;29:191-201.

6. Meyer zum Gottesberge AM, Felix H. Abnormal basement membrane in the inner ear and the kidney of the Mpv17-/- mouse strain: ultrastructural and immunohistochemical investigations. Histochem Cell Biol 2005;124:507-516.

7. Raphael $Y$, Altschuler RA. Structure and innervation of the cochlea. Brain Res Bull 2003;60:397-422.

8. Jahnke K. The fine structure of freeze-fractured intercellular junctions in the guinea pig inner ear. Acta Otolaryngol Suppl 1975;336:1-40.

9. Kikuchi K, Hilding DA. The development of the stria vascularis in the mouse. Acta Otolaryngol 1966;62:277-291.

10. Nakazawa K, Spicer SS, Gratton MA, et al. Localization of actin in basal cells of stria vascularis. Hear Res 1996;96:13-19.

11. Hilding DA, Ginzberg RD. Pigmentation of the stria vascularis. The contribution of neural crest melanocytes. Acta Otolaryngol 1977;84:24-37.

12. Forge $A$, Wright $\mathrm{T}$. The molecular architecture of the inner ear. Br Med Bull 2002;63:5-24.

13. Marcus DC, Wu T, Wangemann $\mathrm{P}$, et al. KCNJ10 (Kir4.1) potassium channel knockout abolishes endocochlear potential. Am J Physiol Cell Physiol 2002;282:C403-C407.

14. Vetter $D E$, Mann JR, Wangemann $P$, et al. Inner ear defects induced by null mutation of the isk gene. Neuron 1996;17:1251-1264.

15. Izzedine H, Tankere F, Launay-Vacher V, et al. Ear and kidney syndromes: molecular versus clinical approach. Kidney Int 2004;65:369-385.

16. White RH, Raafat F, Milford DV, et al. The Alport nephropathy: clinicopathological correlations. Pediatr Nephrol 2005;20:897-903.

17. Cosgrove D, Samuelson G, Meehan DT, et al. Ultrastructural, physiological, and molecular defects in the inner ear of a geneknockout mouse model for autosomal Alport syndrome. Hear Res 1998;121:84-98.

18. Harvey SJ, Mount R, Sado $Y$, et al. The inner ear of dogs with X-linked nephritis provides clues to the pathogenesis of hearing loss in X-linked Alport syndrome. Am J Pathol 2001;159:1097-1104.

19. Vogel WF. Collagen-receptor signaling in health and disease. Eur J Dermatol 2001;11:506-514.

20. Vogel W, Gish GD, Alves F, et al. The discoidin domain receptor tyrosine kinases are activated by collagen. Mol Cell 1997;1:13-23.

21. Shrivastava A, Radziejewski C, Campbell E, et al. An orphan receptor tyrosine kinase family whose members serve as nonintegrin collagen receptors. Mol Cell 1997;1:25-34.

22. Vogel WF, Abdulhussein R, Ford CE. Sensing extracellular matrix: an update on discoidin domain receptor function. Cell Signal 2006;18:1108-1116.

23. Avivi-Green C, Singal M, Vogel WF. Discoidin domain receptor 1-deficient mice are resistant to bleomycin-induced lung fibrosis. Am J Resp Crit Care Med 2006;174:420-427.

24. Ram $\mathrm{R}$, Lorente $\mathrm{G}$, Nikolich $\mathrm{K}$, et al. Discoidin domain receptor-1a (DDR1a) promotes glioma cell invasion and adhesion in association with matrix metalloproteinase-2. J Neurooncol 2006;76:239-248.
25. Hou G, Vogel W, Bendeck MP. The discoidin domain receptor tyrosine kinase DDR1 in arterial wound repair. J Clin Invest 2001;107: 727-735.

26. Vogel WF, Aszodi A, Alves F, et al. Discoidin domain receptor 1 tyrosine kinase has an essential role in mammary gland development. Mol Cell Biol 2001;21:2906-2917.

27. Faraci-Orf E, McFadden C, Vogel WF. DDR1 signaling is essential to sustain Stat5 function during lactogenesis. J Cell Biochem 2006;97:109-121.

28. Gross O, Beirowski B, Harvey SJ, et al. DDR1-deficient mice show localized subepithelial GBM thickening with focal loss of slit diaphragms and proteinuria. Kidney Int 2004;66:102-111.

29. Flamant M, Placier S, Rodenas A, et al. Discoidin domain receptor 1 null mice are protected against hypertension-induced renal disease. J Am Soc Nephrol 2006;17:3374-3381.

30. Meyer zum Gottesberge AM, Felix $\mathrm{H}$, Reuter $\mathrm{A}$, et al. Ultrastructural and physiological defects in the cochlea of the Mpv17 mouse strain. A comparison between young and old adult animals. Hear Res 2001;156:69-80.

31. Carlisle L, Steel K, Forge A. Endocochlear potential generation is associated with intercellular communication in the stria vascularis: structural analysis in the viable dominant spotting mouse mutant. Cell Tissue Res 1990;262:329-337.

32. Hirose K, Liberman MC. Lateral wall histopathology and endocochlear potential in the noise-damaged mouse cochlea. J Assoc Res Otolaryngol 2003;4:339-352.

33. Spicer SS, Schulte BA. Pathologic changes of presbycusis begin in secondary processes and spread to primary processes of strial marginal cells. Hear Res 2005;205:225-240.

34. Spicer SS, Schulte BA. Novel structures in marginal and intermediate cells presumably relate to functions of apical versus basal strial strata. Hear Res 2005;200:87-101.

35. Ito M, Spicer SS, Schulte BA. Immunohistochemical localization of brain type glucose transporter in mammalian inner ears: comparison of developmental and adult stages. Hear Res 1993;71: 230-238.

36. Agarwal G, Mihai C, Iscru DF. Interaction of discoidin domain receptor 1 with collagen type 1. J Mol Biol 2007;367:443-455.

37. Ferri N, Carragher NO, Raines EW. Role of discoidin domain receptors 1 and 2 in human smooth muscle cell-mediated collagen remodeling: potential implications in atherosclerosis and lymphangioleiomyomatosis. Am J Pathol 2004;164:1575-1585.

38. Curat CA, Vogel WF. Discoidin domain receptor 1 controls growth and adhesion of mesangial cells. J Am Soc Nephrol 2002;13: 2648-2656.

39. Morton CC. Gene discovery in the auditory system using a tissue specific approach. Am J Med Genet A 2004;130:26-28.

40. Tlili A, Mannikko M, Charfedine I, et al. A novel autosomal recessive non-syndromic deafness locus, DFNB66, maps to chromosome 6 p21.2-22.3 in a large Tunisian consanguineous family. Hum Hered 2005;60:123-128.

41. Verstreken M, Claes J, Van de Heyning PH. Osteogenesis imperfecta and hearing loss. Acta Otorhinolaryngol Belg 1996;50:91-98.

42. Van Camp G, Snoeckx RL, Hilgert N, et al. A new autosomal recessive form of Stickler syndrome is caused by a mutation in the COL9A 1 gene. Am J Hum Genet 2006;79:449-457.

43. Alves FR, de A Quintanilha Ribeiro F. Revision about hearing loss in the Alport's syndrome, analyzing the clinical, genetic and bio-molecular aspects. Rev Bras Otorrinolaringol (Engl Ed) 2005;71:813-819.

44. Miner JH, Sanes JR. Molecular and functional defects in kidneys of mice lacking collagen alpha 3(IV): implications for Alport syndrome. J Cell Biol 1996;135:1403-1413.

45. Henson MM, Burridge K, Fitzpatrick $D$, et al. Immunocytochemical localization of contractile and contraction associated proteins in the spiral ligament of the cochlea. Hear Res 1985;20:207-214.

46. Lalwani AK, Goldstein JA, Kelley MJ, et al. Human nonsyndromic hereditary deafness DFNA17 is due to a mutation in nonmuscle myosin MYH9. Am J Hum Genet 2000;67:1121-1128.

47. Heath KE, Campos-Barros A, Toren A, et al. Nonmuscle myosin heavy chain IIA mutations define a spectrum of autosomal dominant macrothrombocytopenias: May-Hegglin anomaly and Fechtner, Sebastian, Epstein, and Alport-like syndromes. Am J Hum Genet 2001;69:1033-1045. 